Erschienen in: Klein, Josef (Hrsg.): Politische Semantik. Bedeutungsanalytische und sprachkritische Beiträge zur politischen Sprachverwendung. -

Opladen: Westdeutscher Verlag, 1989. S. 297-326.

\title{
DIE DOPPELTE WENDE - ZUR VERBINDUNG VON SPRACHE, SPRACHWISSENSCHAFT UND ZEITGEBUNDENER POLITI- SCHER BEWERTUNG AM BEISPIEL DEUTSCH-DEUTSCHER SPRACHDIFFERENZIERUNG
}

Manfred W. Hellmann

1. Vorbemerkung

2. Wechselseitige 'Sprachspaltungs'-Polemiken (50er Jahre)

3. Westdeutsche Betonung sprachlicher Divergenz gegen ostdeutsche Betonung sprachlicher Einheit (60er Jahre)

4. Westdeutsche Betonung sprachlicher Gemeinsamkeit gegen ostdeutsche Betonung sprachlicher Differenz (späte 60er und 70er Jahre)

5. Annäherung der sprachwissenschaftlichen Standpunkte (80er Jahre)

6. Resümee und Ausblick

7. Literatur

\section{Vorbemerkung}

Schon vor ziemlich genau 40 Jahren, nämlich im Jahre 1947, erschienen die ersten schriftlichen Hinweise darauf, daß die politische Aufteilung Deutschlands unter die Siegermächte - die Teilung in eine Ost- und drei Westzonen - sprachliche Veränderungen zur Folge hatte, genauer: Veränderungen im Wortschatz. Es waren sowjetrussische Germanisten, die dies zuerst beobachteten. Ihre Hinweise wurden hier allerdings kaum bekannt. Seitdem ist viel gearbeitet, viel geforscht und viel geschrieben worden in Ost und West, und wir wissen heute zum Teil ganz gut Bescheid über die "sprachlichen Besonderheiten" der beiden deutschen Staaten (so sagt man zurückhaltend heute), die "Divergenzen", die "Differenzen", die "Ost-West-Spezifika", den "Sonderwortschatz der SBZ" (so hieß es früher) oder auch "Parteijargon", denn so vor allem sah man bis in die 2. Hälfte der 60er Jahre hinein die Besonderheiten im Sprachgebrauch der DDR die eigenen Besonderheiten ohnehin übersehend.

Wir wissen also teilweise ganz gut Bescheid. Z.B. über die Unterschiede im Wortschatz der öffentlichen Kommunikation, besonders der Presse, insbesondere in den Bereichen der Politik, Ideologie, 
Institutionen, auch wohl Wirtschaft. Dazu gibt es recht gute Einzeluntersuchungen, auch aus der $\mathrm{DDR}^{1}$, es gibt sogar schon Wörterbücher der sprachlichen DDR-Besonderheiten (diese allerdings nur aus der BRD) ${ }^{2}$. Wir kennen die unterschiedlichen Typen dieser Spezifika, die Schwerpunkte ihrer Entstehung, spezifische Gebrauchsweisen, spezif ische Stilmerkmale, begründet in unterschiedlichen Kommunikationssituationen und -bedingungen. Die Landkarte der Wortschatzbesonderheiten weist zwar manche recht dünn gezeichneten Stellen und einige richtige weiße Stellen auf ${ }^{3}$ - auch bedarf sie stetiger Aktualisierung - aber in Umrissen und in vielen Details ist sie bekannt. Anders steht es mit dem, was hinter den Wörtern und ihrer Bedeutung liegt, also mit den Erfahrungen, den erlebten Alltagswelten, auf die sich Wörter, Äußerungen, Sätze, Texte ja letzten Endes beziehen. Worauf, zum Beispiel, referiert ein so schlichter, gemeinverständlicher, deutscher Satz wie

Ich suche eine Wohnung.

Wer sich etwas auskennt in der DDR und seine eigenen Erfahrungen hierzulande diesbezüglich noch nicht vergessen hat, wird mir zustimmen: Gleich hinter diesem Satz - wenn man ihn denn nicht bloß als Satz, sondern als Aufforderung oder Entschluß zur Lösung eines Problems auffaßt - gleich hinter diesem Satz öffnen sich Welten voller Unterschiede, und selbst die entdeckten Gemeinsamkeiten erweisen sich als trügerisch, z.B. weil andere Bewertungsmaßstäbe gelten ${ }^{4}$.

1 Für die Zeit bis 1975 vgl. dazu M.W. Hellmann (1976) (Hg.): Bibliographie zum öffentlichen Sprachgebrauch in der Bundesrepublik Deutschland und der DDR; für den späteren Zeitraum vgl. Burkard Schaeder (1981): Deutsche Sprache in der BRD und in der DDR. In: Muttersprache, Jg. 91, H. 3/4, S. 198 - 205. Ein knapper Überblick auch bei M. W. Hellmann (1984) in dem Sammelband "Ost-West-Wortschatzvergleiche", darin Bericht Nr. I, S. 22 - 31. Zusammenfassend zu den bisherigen Forschungsergebnissen M.W. Hellmann (1980): Deutsche Sprache in der Bundesrepublik Deutschland und in der Deutschen Demokratischen Republik. In: Lexikon der Germanistischen Linguistik, S. 519 - 527

2 Kinne, M. / Strube-Edelmann, B. (1981): Kleines Wörterbuch des DDR-Wortschatzes, München; Ahrends, M. (1986): Trabbi, Telespargel und Tränenpavillon Das Wörterbuch der DDR-Sprache, München

Auf einige solcher Lücken wird hingewiesen bei M.W. Hellmann (1985): Bemerkungen zur Entwicklung und zur gegenwärtigen Lage des Arbeitsgebietes "Ost-WestSprachdifferenzierung". In: Mitteilungen 11 des Instituts für Deutsche Sprache, Mannheim, S. 86

4 So verknüpfen sich mit dem Ausdruck "Neubauwohnung" für die meisten DDR-Bürger weit positivere Bewertungen als für BRD-Bürger, was auf den vergleichsweise desolaten Zustand sehr vieler Altbauwohnungen in der DDR zurückzuführen ist. 
Dies nur als ein Hinweis auf eine terra incognita, und es gibt deren viele. Aber ich muß hier abbrechen, denn sonst befinde ich mich mit wenigen Schritten in einem ganz anderen Thema, denn es lautet ja nicht "Die sprachlichen Besonderheiten" oder "Die Entwicklung der deutschen Sprache in den beiden deutschen Staaten".

Ich will in meinem Beitrag also weniger auf die sprachliche Differenzierung selbst, als vielmehr auf deren öffentliche und sprachwissenschaftliche Bewertung abheben, die, wie ich hoffe darstellen zu können, manchmal bemerkenswert stark von den Wendungen der Begriffe "Nation/Nationalität", d.h. letztlich von den jeweils vorherrschenden deutschlandpolitischen Vorstellungen, beeinflußt worden ist und wird.

\section{Wechselseitige 'Sprachspaltungs'-Polemiken (50er Jahre)}

Ich erlaube mir, mit einem längeren $\mathrm{Zitat} \mathrm{zu}$ beginnen, und zwar auch deshalb, weil es Jahrzehnte später mehrfach wieder aufgegriffen wurde.

Und alle wissen wir, welche ungeheure Rolle für die Einheit einer Nation die Einheit ihrer Sprache spielt. Sie ist das innigste Band, das ein Volk in der Mannigfaltigkeit seiner Gruppen, Klassen und Parteien zusammenhält. ${ }^{5}$

Genau dieser Gedanke von der Einheit der Sprache bildet den innersten Kern der ungemein reichen Sprachbetrachtungen, die Stalin veröffentlichte. Eine Sprache ist nach Herkunft und entscheidender Aufgabe das gemeinsame Eigentum aller Teile der Nation und das gemeinsame Verkehrs- und Verständigungsmittel all ihrer Teile [...] Somit muB es das ideale Ziel der amerikanischen Gewaltherren und des Klüngels ihrer westdeutschen Helfershelfer sein, diese Gemeinsamkeiten aufzuheben, indem nicht nur das neue Sprachgut, der neue Sprachstil des Ostens abgewehrt, sondern zugleich auch die eigene Sprache, der eigene Sprachstil in entgegengesetzte Richtung "fortentwickelt" wird.

Da nun die Einheit der deutschen Nation aufs schwerste gefährdet ist und da alles darauf ankommt, daB ihr geistiger Zusammenhang, ihr einander Verstehen unbedingt gewahrt bleibt, so bedeutet schon die leiseste sprachliche Dissonanz eine schwere Gefahr. ${ }^{6}$

5 Klemperer, V. (1955): Verantwortung für die Sprache. In: Neue Deutsche Literatur, Jg. 3, H. 3, S. 122-126. Vgl. vom selben Verfasser (1952): Unsere Sprache - Ein einigendes Band der Nation. In: Die neue Schule, Jg. 8, S. 4 - 5

6

Klemperer, V. (1954): Zur gegenwärtigen Sprachsituation in Deutschland, Berlin (Ost), 1. Aufl. 1952, S. 10 
Dieser Text mutet nach Inhalt und Wortwahl so altväterlich an, er ist so getragen von einem - ich möchte sagen: nationalen patriotischen Eifer, daß er gewiß manchem Konservativen in der Bundesrepublik zuzutrauen wäre, wohl kaum einem Nationalpreisträger und Volkskammerabgeordneten der DDR - wäre da nicht jener ausdrückliche Bezug auf Stalin und die "amerikanischen Gewaltherren". Das Zitat stammt von Victor Klemperer, dem Verfasser jenes verdienten Buches "LTI- Lingua Tertii Imperii", in dem sich Klemperer in Form von Tagebucheintragungen mit den erlebten und erlittenen Erscheinungen des Nazijargons auseinandersetzt ${ }^{7}$. Klemperer wurde prominent, und er fand Mitstreiter, vor allem F.C. Weiskopf ${ }^{8}$ und später Henrik Becker ${ }^{9}$, die, wie er, sich leidenschaftlich für die Einheit und Reinheit der deutschen Sprache einsetzten, wobei sie sich ebenso gegen die (wie es hieß) "angloamerikanische Überfremdung" und die "Nazirelikte" im Sprachgebrauch der Bundesrepublik wandten, wie auch - und besonders Klemperer - gegen das "Kaderwelsch", den "Funktionärsjargon" der eigenen Funktionäre - auch dieses Stichwort stand bei Stalin:

\begin{abstract}
Läßt sich eine Gruppe in törichter Überheblichkeit dazu verführen, die überkommene Allgemeinsprache ihres Volkes zu mißachten und das Besondere und Neue der eigenen Ausdrucksweise götzenhaft als Sonder- oder Klassensprache zu verehren, dann verkümmert dieses Sprachwesen (oder richtiger: -unwesen) notwendigerweise zum Jargon, und ebenso notwendigerweise beraubt sich die solchem Irrtum verfallene Gruppe der Möglichkeit, an der Fortbildung der nationalen Sprache [...] mitzuwirken. 10
\end{abstract}

Seit 1955 spätestens verstummten derartige Stimmen allerdings in der DDR für lange Zeit.

Auch in der Bundesrepublik konnte man sich über Mangel an Leidenschaftlichkeit in der Auseinandersetzung um das Ost-WestSprachproblem wahrlich nicht beklagen, und der Tonfall der Auseinandersetzungen nahm nach dem Arbeiteraufstand vom 17. Juni 1953 an Schärfe noch erheblich zu. Selbstkritik oder auch nur die

7

Klemperer, V. (1969): "LTI" - Die unbewältigte Sprache. Aus dem Notizbuch eines Philologen. dtv (3.Aufl.) Das Buch ist auch als Reclam-Taschenbuch in beiden deutschen Staaten wieder verfügbar

8

Weiskopf, F. C. (1955): "Ostdeutsch" und "Westdeutsch" oder über die Gefahr der Sprachentfremdung. In: Neue Deutsche Literatur 3/7, S. 79 - 88

9 Becker, H. (1956): Sieben Sprachbriefe zur Gegenwart. Eine gesellschaftliche Sprachund Denklehre, Halle

Klemperer, V. (1954): Zur gegenwärtigen Sprachsituation in Deutschland, S. $7 f$. 
Bereitschaft, auch im eigenen Land die massiven sprachlichen Veränderungen der Nachkriegszeit angemessen zu berücksichtigen, war allerdings nicht Sache der meisten Autoren. Schon die Titel mancher Aufsätze sprechen für sich.

- $\quad$ "Deutsche Sprache in östlicher Zwangsjacke (1954) ${ }^{11}$

- "Sowjetdeutsch' - Die Sprache als Opfer und Werkzeug der Sowjetisierung" (1955) ${ }^{12}$

- $\quad$ "Die Sprachentartung in der Sowjetzone" (1952) ${ }^{13}$

- "Gefährliches 'Parteichinesisch'- Verhängnisvolle Sprachspaltung zwischen West und Ost" (1956) ${ }^{\mathbf{1 4}}$

- $\quad$ "Die Sprache als Waffe" (1958)

- "Sowjet-Deutsch' - Die Sprache als politisches Kampfmitttel der Kommunisten" (1959) ${ }^{16}$

Auch in offiziösen Veröffentlichungen wie z.B. in dem Nachschlagewerk "SBZ von A - Z" des Gesamtdeutschen Ministeriums in Bonn wird die Verantwortung für die eingetretenen sprachlichen Unterschiede als "offizieller Jargon des Regimes" einseitig dem DDR-System zugeschoben:

Die Sprache unseres Volkes ist hüben und drüben die gleiche geblieben. Aber über diese Einheit legt sich in der Sowjetzone die diktatorische Terminologie der alleinherrschenden Partei. Die Sprache der sowjetzonalen Öfentlichkeit ist vom Kauderwelsch des kommunistischen Regimes von heute in einem Maße überfremdet, daß sie dem westdeutschen Leser kaum ohne ein Wörterbuch verständlich ist. Dieses Buch soll deshalb zugleich ein Wörterbuch sein, das den offiziellen Jargon des Regimes in der Sowjetzone übersetzt. ${ }^{17}$

11 Köhler, A. (1954): Deutsche Sprache in östlicher Zwangsjacke. (= Vortrag im deutschen Sprachverein Berlin am 4.12.53), Berlin (West)

12 Koepp, F. (1955): 'Sowjetdeutsch'- Die Sprache als Opfer und Werkzeug der Sowjetisierung. In: Akademische Blätter 57/3, S. 41 - 46

13 Borée, K.F. (1952): Die Sprachentartung in der Sowjetzone. In: PZ-Archiv 3/2, S. 23 $-24$

14 Haefs, H. (1956): Gefährliches "Parteichinesisch" - Verhängnisvolle Sprachspaltung zwischen West und Ost. In: Das Parlament 15.8.1956, S. 9

15 Herrmann, E.M. (1958): Die Sprache als Waffe. In: Hessische Blätter für Volksbildung $8 / 3$, S. $135-140$

Korntner, B. (1959): 'Sowjet-Deutsch' - Die Sprache als politisches Kampfmittel der Kommunisten. In: Die Orientierung (Pfaffenhofen), Beiheft Nr. 12, o. J.

SBZ von A bis Z (1954). Hrsg. vom Bundesministerium für gesamtdeutsche Fragen, 2. Aufl. (Vorwort). Ähnlich auch noch in der 8. Auflage von 1963 unter dem Stichwort "Sprache", jedoch mit einer Distanzierung vom Ausdruck "Sprachspaltung" 
Den Vogel schoß zweifellos Otto B. Roegele ab - später Ordinarius für Publizistikwissenschaften in München -, der 1959 in der Zeitschrift "Die politische Meinung" von einem "System des kommunistischen Sprachkrieges" sprach, von der "Fälschung der Begriffe" und der "Schaffung einer eigenen Sprachwelt", von "thematischer Verführung" usw., und dazu meinte:

Das ist der teuflisch-grandiose Versuch, durch Umschaffung der Worte auch das Weltbild der Menschen umzuschaffen, der Versuch, die zweite Schöpfung [...] zu pervertieren in die satanische Schöpfung einer Gegenwelt [...] "Gegenschöpfung ist immer Mord, nämlich Aneignung durch gewaltsame Zerstörung". Das gilt auch hier, gerade hier. [S. 48]

Innerhalb dieser [ $=$ der kommunistischen, d.V.] Terminologie haftet der Bezeichnung "Deutsche Demokratische Republik" auch nicht jener Geschmack des Widernatürlichen an, den jeder gesund Empfindende wahrnehmen muß [...] Gibt es irgendeinen Grund zu der Hoffnung, daß die Menschen dieser Vergewaltigung der Gedanken durch die Worte widerstehen können? Es spricht einiges dafür, daß die Menschen, ungeachtet der gnadenlosen Konsequenz der Propaganda-Maschine, ihr gesundes Sprachempfinden bewahrt haben. [S. 60] $]^{18}$

Das ist - schon damals - "das Reich des Bösen".

3. Westdeutsche Betonung sprachlicher Divergenz gegen ostdeutsche Betonung sprachlicher Einheit

Besonnene Stimmen konnten sich in diesem Klima des kalten Krieges nur langsam Gehör verschaffen oder blieben zunächst vereinzelt:

Die politische Abtrennung hat in den wenigen Jahren nach dem Kriege in Deutschland eine tiefe sprachliche Kluft entstehen lassen. Die deutsche Sprache in der sowjetischen Besatzungszone (SBZ) erlitt in Folge der politischen Verhältnisse starke Veränderungen [...]

Bedeutsamer als alle Neubildungen sind [...] die Änderungen der Wortbedeutung, ist die Wandlung der Begriffe, die sich in der DDR vollzogen hat.

Die $\mathrm{Zahl}$ all jener sprachlichen Veränderungen ist, am Gesamtbestand der Sprache gemessen, natürlich gering [...] Aber die neue Sprache beherrscht immerhin den gesamten Bereich des gedruckten Wortes, ... sie ist die Sprache des öffentlichen Lebens im weitesten Sinne.

Die Gefahr einer propagandistischen Normierung ist auch im Westen gegeben; an die Stelle einer nüchternen, immer von neuem unternommenen Prüfung der Tatsachen können leicht schablonenhafte Vorstellungen treten $[\ldots]$

18 Roegele, O.B. (1959): Die Spaltung der Sprache. Das kommunistische Deutsch als Führungsmittel. In: Die politische Meinung 4/36, S. $48-60$ 
[...], daß die totalitären Möglichkeiten der Sprache auch in Westdeutschland $[. .$.$] gegeben sind, daB ihre Gefahren aber durch die bloBe Existenz$ des (echten) formalen Prinzips der Demokratie (Mehrstimmigkeit) gemildert werden. ${ }^{19}$

Dieser relativ nüchterne Aufsatz aus dem Jahre 1953 von Walter Richter (Pseudonym für den in der Schweiz lebenden Germanisten Peter Brang) wurde erst Ende der 50er Jahre wiederentdeckt, als sich gleichzeitig drei Hochschulgermanisten in nun endlich wissenschaftlicher Weise mit dem Thema befaßten: Gustav Korlén in Schweden ${ }^{20}$, Werner Betz in München ${ }^{21}$ und Hugo Moser in Bonn ${ }^{22}$. Werner Betz vor allem ist es zu verdanken, daß die These einer "Sprachspaltung" jedenfalls in der Linguistik niemals mehr wiederholt wurde. Auch Hugo Moser, der anfangs die "Gefahr einer sprachlichen Spaltung" noch sah, korrigierte sich und sprach von der Gefahr einer sprachlichen "Sonderung":

Es hat im Zusammenhang mit der politischen Teilung Deutschlands seit 1945 eine entgegengesetzte Entwicklung eingesetzt, die von manchen drüben und auch von einigen hüben als "Sprachspaltung" bezeichnet wird [...] Ich wende mich gegen diese Bezeichnung aus Gründen, die ich schon an anderer Stelle dargelegt habe. Gemeinsam sind der Sprache in beiden deutschen Staaten weiterhin nicht bloB die Normen der Rechtschreibung und der Hochlautung, sondern vor allem auch der allergröBte Teil des Wortschatzes einschließlich der Wortinhalte und so gut wie die ganze Struktur des Satzbaus einschlieBlich der Flexionsformen. Wohl aber muB man von der Gefahr einer neuen Sonderung sprechen. ${ }^{23}$

Kann und darf man nicht von einer Sprachspaltung sprechen, so besteht aber ohne Zweifel die Gefahr, daB, besonders in gewissen zentralen Bereichen des Wortschatzes, namentlich bei den Wortinhalten, früher oder

19 Richter, W. (1953): Zur Entwicklung der deutschen Sprache in der sowjetischen Besatzungszone. In: Europa-Archiv 8/21, S. 6053 - 6056

Korlén, G. (1959): Zur Entwicklung der deutschen Sprache diesseits und jenseits des Eisernen Vorhangs. In: Deutschunterricht für Ausländer, Jg. 9, S. 138 - 153

21 Betz, W. (1962): Zwei Sprachen in Deutschland? In: Merkur 16/9, S. 873 - 879. Nachdruck dieses Aufsatzes in dem Sammelband von Handt (Hg.) (1964): Deutsch Gefrorene Sprache in einem gefrorenen Land?

22 Moser, H. (1961): Die Sprache im geteilten Deutschland. In: Wirkendes Wort 11/1, S. 1 - 21; ders. (1962): Sprachliche Folgen der politischen Teilung Deutschlands. (= Beiheft zum 'Wirkenden Wort', Nr 3), Düsseldorf

Moser, H. (Hg.) (1964): Das Aueler Protokoll - Deutsche Sprache im Spannungsfeld zwischen Ost und West. Düsseldorf (aus dem Vorwort) 
später eine tiefer reichende Auseinanderentwicklung sprachimmanenter Art eintreten kann. ${ }^{24}$

Das gleiche Schlagwort "Sprachspaltung" diente allerdings noch für Jahre als Zündstoff für Polemiken der DDR-Linguisten gegen die der BRD.

Andere Germanisten sprachen von Divergenzen, Differenzierungserscheinungen oder allgemein von der "Sonderentwicklung der DDR"- woraus ersichtlich wird, daß auch weiterhin die Besonderheiten der DDR bei weitem im Mittelpunkt des Interesses standen, und auch hier wieder vor allem die Wörter mit politisch-ideologischen Implikationen. $\mathrm{Da} \beta$ es Wortschatzveränderungen auch im DDR-Alltag gab - ebenso wie in dem der BRD - wurde nur äußerst selten wahrgenommen. ${ }^{25}$

Die Absperrmaßnahmen vom 13. August 1961 hatten noch einmal eine Flut heftiger Angriffe auf die Spaltungsbemühungen der SED zur Folge, die nunmehr, wie man befürchtete, endgültig auch in der Sprache durchgesetzt werden sollten. Solche Polemiken flackerten in der Presse der Bundesrepublik, allerdings zunehmend beschränkt auf die Rechtspresse, noch bis Ende der 60er Jahre immer wieder auf. Auch die Germanistik/Linguistik in der BRD blieb davon nicht immer verschont, wie einige Beiträge aus dem sonst oft $\mathrm{zu}$ pauschal kritisierten "Aueler Protokoll" belegen. ${ }^{26}$

Im großen und ganzen machte sich jedoch die Sprachgermanistik in der Bundesrepublik - trotz oder gerade wegen der Ernüchterung nach dem Mauerbau, auf den Weg zu einer mehr nüchternen, empirisch fundierten, auch die Erscheinungen in der Bundesrepublik mit berücksichtigenden Betrachtungs- und Vorgehensweise. Am deutlichsten wurde dies in dem - wie er selbst ironisch sagte -, "rein destruktiven" Aufsatz von Walther Dieckmann "Kritische Bemerkungen zum sprachlichen Ost-West-Problem" (1967), in dem er einem Großteil der bis dahin publizierten westdeutschen Literatur eine ganze

24

Moser, H. (Hg.) (1964): Das Aueler Protokoll - ...(aus dem Umschlagtext)

Zu den Ausnahmen gehört der Versuch von Hugo Moser (1971): Zum Problem der "neutralen" Neuprägungen und Neubedeutungen im offiziellen Sprachgebrauch der DDR. In: Schwenk, S. (Hg.) u. a.: Et multum et multa - Festgabe für Kurt Lindner, Berlin / New York, S. 249-255

Zu den meist kritisierten Aufsätzen gehört der von Hannes Maeder (1964): Sprache und Totalitarismus. In Moser, H. (Hg.) (1964): Das Aueler Protokoll - ..., S. 13 - 23; daneben auch der Aufsatz von Ernst Riemschneider und Hansjürgen Schierbaum im gleichen Sammelband 
Reihe methodischer Mängel sowie politische Voreingenommenheit vorhielt.

Statt einer sachlichen empirischen Beschreibung und einer sorgfältigen
Bestandsaufnahme spielen von der Einleitung bis zum SchluB sprachtheo-
retische Prämissen und politisch-ideologische Standpunkte in die Argu-
mentation hinein, wobei ungewiB nur ist, ob die letzteren die ersteren
stützen sollen oder umgekehrt. Beide verbinden sich widersprüchlich zu
einem gemeinsamen Ziel [...].
Das sprachwissenschaftliche Interesse ist ungleichmäBig entwickelt. Die
Probleme, die die Sprache der Politik und auch die spezielle Thematik des
sprachlichen Ost-West-Gegensatzes bieten, sind zum Teil noch gar nicht
in Angriff genommen worden. Wo sie diskutiert werden, bleibt man bei
vagen Vorläufigkeiten stehen. [...] Die wesentlichen Fragen sind noch un-
gelöst und manchmal noch gar nicht erkannt. Vertraut man den Einlei-
tungen und SchluBworten, so ist das eigentliche "Anliegen" eher ein poli-
tisches, ein menschliches, ein ethisches als ein sprachwissenschaftliches;
$[\ldots]^{27}$

Dieckmanns Kritik war im Einzelfall gewiß überzogen, im Prinzip jedoch berechtigt, in mancher Beziehung geradezu ein Durchbruch. $\mathrm{Da}$ der Aufsatz so wirksam werden konnte, lag sicher auch am politischen Klima jener Jahre, in denen sich eine ganze akademische Generation aus den Fesseln des kalten Krieges zu befreien versuchte. Ich greife wieder zurück:

Die DDR-Germanistik meldet sich erst 1963/64 wieder zu Wort. Karl-Heinz Ihlenburg ${ }^{28}$ und auch Hans-Joachim Gernentz ${ }^{29}$ stellten in ihren Aufsätzen zahlreiche Besonderheiten der DDR vor und verteidigten sie als fortschrittlich, zukunftsweisend und auch als in vieler Hinsicht unvermeidlich. Keineswegs, so heißt es übereinstimmend in den Arbeiten dieser Zeit, werde dadurch die Einheit der deutschen Sprache gefährdet, im Gegenteil werde sie, und zwar gerade in der DDR, progressiv und im Sinne einer geistigen Erneuerung weiterentwickelt. Nicht die "westdeutsche Bundesrepublik", die sich vom Nationalverband abgespalten habe, sondern die DDR sei der ei-

27 Dieckmann, W. (1967): Kritische Bemerkungen zum sprachlichen Ost-West-Problem. In: Zeitschrift für deutsche Sprache 23/3, S. 136 - 165 (hier S. 164 und 165)

Ihlenburg, K.-H. (1964): Entwicklungstendenzen des Wortschatzes in beiden deutschen Staaten. In: Weimarer Beiträge 10/3, S. 372 - 397

Gernentz, H.-J. (1965): Droht dem Deutschen die Gefahr einer Spaltung in zwei Sprachen? In: jezyky obce w szkole, Fremdsprachen in der Schule (Warschau), 2/2, S. 69 - 80; ders. (1967): Zum Problem der Differenzierung der deutschen Sprache in beiden deutschen Staaten. In: Weimarer Beiträge 13/3, S. $463-468$ 
gentliche Hort der deutschen Nationalsprache. Besonders klar wird dies in dem Aufsatz von Joachim Hoeppner: 'Widerspruch aus Weimar' (1964), der in dem westdeutschen Sammelband "Deutsch - Gefrorene Sprache in einem gefrorenen Land?" veröffentlicht ist. Besonders aufschlußreich auch deshalb, weil Hoeppner dort unmittelbar auf die Äußerungen des Schweden Gustav Korlén ${ }^{30}$ antwortet.

Selbstverständlich wird in beiden Teilen Deutschlands nach wie vor deutsch gesprochen und der grundlegende Wortbestand ist derselbe. Dennoch wurden in der DDR, aber im beträchtlichen Maße auch in Westdeutschland, nicht nur neue Bezeichnungen für alle Bereiche des gesellschaftlichen Lebens geprägt, sondern vor allem viele geläufige Wörter mit neuem begrifflichen Inhalt erfüllt, der ihre herkömmliche Bedeutung wandelte.

[...] Sicher geht es nun zu weit, von einer "Spaltung der Sprache" zu sprechen, wie das in Westdeutschland manchmal geschieht, um die uns angeblich zufallende Schuld desto schwerer zu wägen und unser Bemühen um Verständigung von vornherein abzuwehren. Die deutsche Sprache ist nach wie vor gemeinsames nationales Verständigungsmittel, und auch dort, wo es um die stärksten Bedeutungsunterschiede geht, bedienen wir uns ihrer.

[...] Korlén fürchtet, wir spalteten mit unseren Neuwörtern und Neubedeutungen die deutsche Sprache. Diese Behauptung [...] ruft auch bei einigen unserer Germanisten Unsicherheit hervor. Oft wird sie nur vorgebracht, um mit der Abneigung gegen diesen Wortschatz und seinen Sinngehalt auch die Ablehnung der Sache zu wecken, die er ausdrückt.

Die Einheit der deutschen Sprache [...] kann [...] nicht darin bestehen, den alten, einseitigen oder falschen, unzulänglich und oft unwahr gewordenen bürgerlichen Sinngehalt zu verteidigen oder unaufrichtig hinter "Neuschöpfungen" zu verbergen, auch nicht darin, neue und alte Bedeutung zu vereinen, einander anzugleichen und als gleichberechtigt gelten zu lassen. Gerade in diesem von Ideologie erfülten Bereich der Sprache ist das heutige Nebeneinander und Gegeneinander historisch ein Nacheinander, in dem nur das dem neuen Leben angemessene eine Zukunft hat. Die Einheit der Sprache beruht auf der Einheit der Denkweise, diese auf der Einheit der Lebensweise, sie alle haben in unserer Zeit ihre Heimat in der sozialistischen Nation. ${ }^{31}$

Deutschlandpolitisch dominierte in der DDR in jener Zeit (bis 1966) weiterhin das Bemühen, durch immer erneute Vorschläge zur Wie-

30 Korlén, G. (1964): Zur Entwicklung der deutschen Sprache diesseits und jenseits des eisernen Vorhangs. In Handt, Fr. (Hg.) (1964): Deutsch - Gefrorene Sprache ..., S. 123 - 142; ders. im gleichen Sammelband: Nachtrag zu Joachim Hoeppner (S. 152 154

Hoeppner, J. (1964): Widerspruch aus Weimar. In: Handt, Fr. (Hg.) (1964): Deutsch

- Gefrorene Sprache ..., S. 143 - 151 
derherstellung der deutschen Einheit auf die Bundesrepublik und die Westmächte Einfluß zu nehmen. Vorschläge für einen Friedensvertrag, Vorschläge für eine Konföderation der beiden deutschen Staaten, zur Einrichtung gemeinsamer Kommissionen lösten einander $a b$, flankiert gelegentlich von Aktionen wie "Deutsche an einen Tisch" oder "Das offene deutsche Gespräch", die alle von der Grundposition ausgingen: Die DDR will die Einheit - freilich zu ihren Bedingungen.

$\mathrm{Zu}$ dieser politischen Linie paßt die Intention eines Mitte der 60er Jahre konzipierten, von dem Ostberliner Sprachwissenschaftler J. Scharnhorst herausgegebenen Sammelbandes ("Sprache in beiden deutschen Staaten"), in dem die Einheit der deutschen Sprache betont wird. Auf diesen Band, der Ende der 60er Jahre erscheinen sollte, jedoch nie ausgeliefert wurde, werde ich im nächsten Kapitel zurückkommen.

4. Westdeutsche Betonung sprachlicher Gemeinsamkeit gegen ostdeutsche Betonung sprachlicher Differenz (späte 60er und 70er Jahre)

Seit 1967 ändert sich das Bild in gleitenden Übergängen. In der BRD hat sich die Große Koalition gebildet, es beginnen die Verhandlungen mit den osteuropäischen Nachbarstaaten und der UdSSR, die von der DDR als eine abgefeimte Umgehungsstrategie empfunden und als ganz besonders gefährlicher Einmischungsversuch der "westdeutschen Imperialisten und Revanchisten" gebrandmarkt wird. Die DDR schon unter Ulbricht - und nicht erst unter Honecker - begibt sich ab 1967/68 auf Abgrenzungskurs. Manipulationsvorwürfe nehmen an Schärfe zu, wie der Sammelband von Günther Heyden "Manipulation" ${ }^{\text {32 }}$ zeigt. Dort ist von der "Fratze der Unmenschlichkeit" (das Bonner System) die Rede, von "Mißbrauch der Sprache", von "Täuschung", "Diffamierung" und anderen polemischen Beschuldigungen aus dem Arsenal des Kalten Krieges. Die Formel "Nichts verbindet uns mit der imperialistischen Bundesrepublik - alles mit der DDR, unserem sozialistischen Vaterland!" stammt schon aus dieser Zeit. Die Propaganda wird nachhaltig umgestellt: Nicht mehr der "Weg zur Wiederherstellung der deutschen Einheit" (unter sozialistischen Vor-

32 Heyden, G. (Hg.) (1968): Manipulation. Die staatsmonopolistische Bewußtseinsindustrie. Berlin (Ost) 
zeichen) ist das Ziel, sondern die Herausbildung der "sozialistischen Menschengemeinschaft", der "sozialistischen Nation" in einer "sozialistischen Staatengemeinschaft". Die völkerrechtliche Anerkennung wird das oberste außenpolitische Ziel der DDR.

In gleichem Maße, wie diese Politik der Selbständigkeit, der Unabhängigkeit, der eigenen nationalen Zukunft in der DDR sich Geltung verschafft, wird in den Medien der BRD ein bezeichnender Tendenzumschwung erkennbar. Während vorher eher vereinzelt auf die verbindende Kraft und Funktionsfähigkeit der deutschen Sprache hingewiesen wird, übernehmen diese Einstellung jetzt auch Blätter, die man eher dem rechten Spektrum zuordnen möchte. Man konstatiert, daß sich die verbindende Kraft der deutschen Sprache sowie vor allem der Widerwillen der Bevölkerung in der "DDR" gegen den Jargon des Systems gemeinsam als stärker erwiesen hätten als alle Sprachspaltungsversuche, die man doch vorher als so gefährlich erfolgreich angeprangert hatte.

In der Politik der 1969 neugebildeten sozialliberalen Koalition wird nun politisches Programm, was sich in der Großen Koalition seit 1967 schon angebahnt hatte: Abbau von Spannungen gegenüber den osteuropäischen Staaten und der Sowjetunion, mehr Kontakte und "menschliche Erleichterungen", Abschluß von Verträgen auf den Gebieten gemeinsamen Interesses mit der DDR. Walter Ulbricht unterbreitete am 18./19. Dezember 1969 einen Vertragsentwurf zum Abschluß völkerrechtlicher Beziehungen zwischen der Bundesrepublik Deutschland und der Deutschen Demokratischen Republik ${ }^{33}$ oder auch, wie es hier in der DDR zum erstenmal heißt, "zwischen der DDR und der BRD" (die Abkürzung "BRD" war bis dahin in der DDR verboten). Willy Brandt antwortete darauf im Januar in seinem Bericht zur Lage der Nation:

25 Jahre nach der bedingungslosen Kapitulation des Hitlerreiches bildet der Begriff der Nation das Band um das gespaltene Deutschland. Im Begriff der Nation sind geschichtliche Wirklichkeit und politischer Wille vereint. Nation umfaBt und bedeutet mehr als gemeinsame Sprache und Kultur, als Staat- und Gesellschaftsordnung. Die Nation gründet sich auf das fortdauernde Zusammengehörigkeitsgefühl der Menschen eines Volkes.

Niemand kann leugnen, daB es in diesem Sinne eine deutsche Nation gibt und geben wird, soweit wir voraus zu denken vermögen. Im übrigen: Auch

33

Neues Deutschland vom 18./19. Dezember 1969, S. 1: Entwurf eines Vertrages über die Herstellung völkerrechtlicher Beziehungen zwischen der Deutschen Demokratischen Republik und der Bundesrepublik Deutschland 
oder, wenn man so will, selbst die DDR bekennt sich in ihrer Verfassung als Teil dieser deutschen Nation. ${ }^{34}$

Dies war für beide deutsche Staaten der Beginn eines langen Weges über Erfurt und Kassel aufeinander zu oder zumindest nebeneinander her, der noch lange nicht beendet ist.

Die DDR sicherte diesen Weg innenpolitisch mit einem Kurs verschärfter Abgrenzung $\mathrm{ab}$, der gerade die Bereiche besonders betraf, die in der Bundesrepublik als noch gemeinsam oder wieder zu stärken hervorgehoben worden waren: Sprache, Kultur, menschliche Kontakte, das Bewußtsein, doch ein Volk zu sein. Dazu Walter U1bricht im Juni 1970:

\begin{abstract}
Sogar die einstige Gemeinsamkeit der Sprache ist in Auflösung begriffen. Zwischen der traditionellen deutschen Sprache Goethes, Schillers, Lessings, Marx und Engels, die vom Humanismus erfüllt ist, und der vom Imperialismus verseuchten und von den kapitalistischen Monopolverlagen manipulierten Sprache in manchen Kreisen der westdeutschen Bundesrepublik besteht eine große Differenz. Sogar gleiche Worte haben oftmals nicht mehr die gleiche Bedeutung [...] Wenn wir z. B. von Gleichberechtigung und Nicht-Diskriminierung sprechen, dann meinen wir eben echte Gleichberechtigung und Nicht-Diskriminierung. Wenn jedoch manche politische Führer in Bonn von Gleichberechtigung sprechen, dann verstehen sie darunter Unterwerfung der DDR. Und wenn sie Nicht-Diskriminierung predigieren, dann meinen sie Verewigung der Diskriminierung der DDR und ihrer Bürger [...] Vor allem aber müssen wir feststellen: Die Sprache der Hitlergenerale, der Neonazis und der Revanchepolitiker gehört nicht zu unserer deutschen Sprache, zur Sprache der friedliebenden Bürger der Deutschen Demokratischen Republik, die wir lieben, schätzen und weiterentwickeln. 35
\end{abstract}

Dieser zwar vorbereitete, aber in seiner Schärfe doch überraschende Kurswechsel hatte für die Linguistik in der DDR eine unmittelbare und mehrere mittelbare Folgen.

Die unmittelbare: Ein lange angekündigter, schon fertig gedruckter Sammelband von J. Scharnhorst (als Herausgeber) verschwand nahezu spurlos. Der Band mit dem Titel "Sprache in beiden deutschen Staaten - Beiträge zum Thema Sprache und Politik" sollte die erste Buchveröffentlichung des 1968 neu gegründeten (besser: umgegründeten) Zentralinstituts für Sprachwissenschaft in Ost-Berlin sein, das als prominentes Akademie-Institut nicht nur personell her-

Brandt, W. (1970): Zur Lage der Nation, Bonn, S. 5

Ulbricht, W. (1970): Rede auf der 13. Tagung des ZK der SED. In: Neues Deutschland Jg. 25, Nr. 164, 16. Juni 1970, S. 4 
vorragend ausgestattet war, sondern auch den Vorstellungen der SED von gesellschaftlicher Wirksamkeit von Sprache - und der Sprachwissenschaft - besser entsprechen sollte. Durch Zufall gelangte ein einziges Exemplar dieses nie ausgelieferten Bandes in unsere Bibliothek (des Instituts für deutsche Sprache, Mannheim). Einige der dort enthaltenen Beiträge erschienen in der DDR später an anderer Stelle, andere nicht. Wir haben die Gründe für das Nichterscheinen natürlich in der Gesamtanlage des Bandes einerseits und in den nichterschienenen Beiträgen andererseits gesucht.

Zusammenfassend ergab sich folgendes Bild:

Der Band hat unter anderem das Ziel,

- den westdeutschen Sprachspaltungsvorwurf endgültig ad absurdum zu führen,

- einschlägige westdeutsche Veröffentlichungen (vor allem das sogenannte "Aueler Protokoll") als voreingenommen und unwissenschaftlich zu entlarven,

- das Vokabular der Großen Koalition, insbesondere der Ostpolitik unter Außenminister Willy Brandt, als manipulativ und revanchistisch zu entlarven,

- die eigenen Neuerungen als positiv und fortschrittlich herauszustellen.

Vom Punkt 4 abgesehen ging das so einfach nicht mehr. Wenn Ulbricht die Auflösung der deutschen Sprache konstatiert, kann man nicht gegen Sprachspaltungsvorwürfe die deutsche Spracheinheit verteidigen. Wenn Stoph sich auf den Weg von Erfurt nach Kassel macht, um mit einem Bundeskanzler Willy Brandt zu verhandeln, geraten Polemiken gegen die "imperialistische westdeutsche Bundesrepublik", die inzwischen zur "BRD" avanciert war, ins Zwielicht. Der Band - trotz schöner Einzelbeiträge - lag schief - und er verschwand.

Die indirekten Folgen:

Zunächst ein Blick auf die politische Entwicklung: Nachdem Ulbricht die Macht an Honecker abgegeben hatte, entschloß sich die DDRFührung zu einer Doppelstrategie:

Einerseits zur Politik der Verhandlungen, die 1973 in den Grundlagenvertrag mündeten und zu einer Fülle von weiteren Vereinbarungen führten, d.h. zu einer deutlichen Entspannung, Entkrampfung im deutsch-deutschen Verhältnis. Dies brachte der SED einen schönen Erfolg (u.a.): die internationale Anerkennung, auch in der UNO. 
Zum anderen zur Politik der Abgrenzung: Die "Nichts-verbindetuns"-Parole der Ulbricht-Zeit mündete in die These von der sozialistischen Nation in der DDR, die sich offensiv abgrenzt von der kapitalistischen Nation in der BRD. Da man ja nicht ganz leugnen konnte und wollte, doch irgendwie auch deutsch zu sein, wählte man folgenden Ausweg: Alle historisch gemeinsamen ethnischen Merkmale (Sprache, Kultur, Geschichte, Sitten und Gebräuche) wies man dem Begriff "Nationalität" zu; alles jedoch, was eine Gesellschaft "wesentlich" kennzeichnet - z.B. Produktionsverhältnisse, Gesellschaftstruktur, Wirtschaftssystem, politisches System, Rechtswesen - dem Begriff "Nation". Mit zunehmender Entwicklung zum Sozialismus werden, so heißt es, die ethnischen Merkmale eher unwesentlich und der Abstand zur BRD somit größer, die Gemeinsamkeit mit den sozialistischen Bruderländern umso intensiver. Während es im Bericht der Bundesregierung 1972 heißt:

Die Deutschen in ihrer Gesamtheit sind in unseren Jahren keine Staatsnation, sie sind dennoch durch viel mehr als bloB die gemeinsame Sprache verbunden. Im menschlichen Bereich noch immer durch unzählige familiäre Bande, im geistigen durch eine gemeinsame Geschichte und Literatur. $\mathrm{DaB}$ sich dies nicht ändert, bis in der Zukunft eine politische Verbindung möglich sein wird, dazu bedarf es jener Politik, die die Bewahrung der Nation erstrebt. ${ }^{36}$

- erklärt dazu Erich Honecker 1973:

Nicht Sprache und Kultur haben die Grenze zwischen der DDR und der BRD gezogen, sondern die unterschiedliche, ja gegensätzliche Struktur der DDR und der BRD [...]

Gemeinsamkeiten in der Sprache können diese Unterschiede nicht hinwegzaubern. ${ }^{37}$

Auch in der neuen DDR-Verfassung von 1974 fand die neue Auffassung Eingang: Während es in der sogenannten Ulbricht-Verfassung von 1968 noch hieß: "Die Deutsche Demokratische Republik ist ein sozialistischer Staat deutscher Nation", heißt es in der Neufassung: "Die Deutsche Demokratische Republik ist ein sozialistischer Staat der Arbeiter und Bauern".

Bezogen auf die Sprache formuliert Siegbert Kahn in der "Weltbühne" 1974 das neue Programm folgendermaßen:

36 Bundesministerium für innerdeutsche Beziehungen (1972): Bericht der Bundesregierung und Materialien zur Lage der Nation, Bonn, S. VIII - IX

Honecker, E. (1973): Rede auf der 9. Tagung des Zentralkomitees 1973. In: Neues Deutschland vom 29.5.1973 
Es ist nicht zu bezweifeln, daB die Sprache eines der Merkmale der Nation und natürlich auch der sozialistischen Nation ist. Und in den vergangenen 25 Jahren ist eine bedeutende Differenzierung der Sprache in der DDR und in der BRD eingetreten [...]

Es handelt sich um eine hochpolitische Sache: Die sozialistische Nation kämpft um ihre unverfälschte Nationalsprache, sie grenzt sich offensiv von der mit Amerikanismen und Anglizismen durchsetzten Sprache ab, die in der imperialistischen BRD gesprochen und geschrieben wird $[. .]$.

Aber die eigentliche Umsetzung für die Linguistik leistete erst Gotthard Lerchner, Linguist an der Universität Halle (ich zitiere hier aus der popularisierten Version in der Zeitschrift Forum 1976):

Zunächst: Das Schlagwort vom "einigenden Band der deutschen Sprache" hat sehr viel oberflächliche Evidenz für sich. Es gehört zur allgemeinen Alltagserfahrung, daB zwischen den Bürgern der DDR und der BRD eine nahezu mühelos einsetzbare sprachliche Kommunikationsfähigkeit besteht. Von zwei verschiedenen Sprachen sprechen zu wollen steht mithin im Widerspruch zu jeder empirischen Erkenntnis [...]

Das Schlagwort vom "einigenden Band der deutschen Sprache" kann zumindest keine nationalsprachliche Einheit mehr reklamieren, sondern sich allenfalls auf Ubereinstimmungen in lediglich einer, nämlich der schriftoder literatursprachlichen Existenzform des Deutschen berufen. Die Wirklichkeit des sprachlichen Gebrauchs ist hier wie dort von der Realität gegensätzlicher Gesellschaftsordnungen bestimmt [...] Wir haben es nunmehr mit vier nationalsprachlichen Varianten zu tun: dem Deutschen in der DDR, der BRD, in Österreich und in der Schweiz. ${ }^{39}$

Dies ist die "Vier-Varianten-These". Sie sollte für nahezu zehn Jahre die Stellungnahmen der DDR-Linguistik, die Vor- und Nachworte der Dissertationen und Tagungsreferate prägen. Nur wenige - darunter Wolfgang Fleischer und Wolfdietrich Hartung - vermieden allzu auffällige Beifallskundgebungen.

In der Bundesrepublik verstärkte sich in den 70er Jahren der Trend, die Gemeinsamkeiten stärker zu betonen - Tenor: Das einigende Band hält. Während Friedrich Luft in der WELT 1958 beim Vergleich zweier DUDEN-Ausgaben noch entsetzt feststellte:

Wir sind soweit. Wir sprechen schon das doppelte Deutsch. Wir haben die feindlichen Duden schon im Haus. ${ }^{40}$

Kahn, S. (1974): Nation und Sprache. In: Die Weltbühne Nr. 53 vom 31.12.74, Berlin (Ost)

Lerchner, G. (1976): Nationalsprachliche Varianten. In: Forum 3, S. 10 - 11

Luft, F. (1958): Wenn das Wörterbuch zur Parteifibel wird. Der Duden links und rechts der Zonengrenze. Wir sprechen schon zweierlei Deutsch. In: DIE WELT vom 5.4.1958 
sah Dittmar in der gleichen Zeitung 1979 gerade das Gegenteil:

Vor allem beweist der Vergleich der beiden Wörterbücher [= des 6-bändigen DUDEN-Wörterbuchs und des Ost-Berliner WDG, d. V:]: Es gibt nur eine deutsche Sprache. ${ }^{4142}$

5. Annäherung der sprachwissenschaftlichen Standpunkte (80er Jahre)

Angesichts der allgemeinen Gemeinsamkeitseuphorie in der Bundesrepublik sahen sich einige Sprachwissenschaftler hier veranlaßt, ein wenig gegenzusteuern: So unproblematisch verlaufe Verständigung wahrlich nicht immer, sprachliche Unterschiede reichten bis tief in den beruflichen und familiären Alltag, und die unterschiedlichen Erfahrunghorizonte seien ein ungeklärtes Problem. Reflexe dieser vorsichtigen Warnungen finden sich auch in den (damals gerade gründlich überarbeiteten) "Materialien zur Lage der Nation":

Die Gleichartigkeit der deutschen Sprache ist unbestritten. Rechtschreibung, Satzbau und Formenlehre zeigen bis heute keine ins Gewicht fallenden Unterschiede in der Bundesrepublik Deutschland und in der DDR. Im Wortschatz sind allerdings, besonders im ideologisch-politischen Bereich, zahlreiche Neuwörter, unterschiedliche Abkürzungswörter und Bedeutungsverschiebungen festzustellen. Inwiefern durch solche Wortschatzdifferenzierungen zwischen Bürgern der Bundesrepublik Deutschland und der DDR Verständigungsprobleme auftreten, ist eine wissenschaftlich bisher kaum untersuchte Frage.

In der Sprachwissenschaft wird heute häufig davon ausgegangen, daB der EinfluB der jeweiligen gesellschaftlichen Werte und Normen auf die Alltagssprache nicht zu unterschätzen sei. Zwischen den Bevölkerungen in beiden deutschen Staaten bestehe heute keine volle Gemeinsamkeit im sprachlichen Medium mehr. Damit seien allerdings grundsätzlich die breiten Möglichkeiten der Information und Kommunikation in Deutschland nicht aufgehoben $[. .$.

41 Dittmar, P. (1979): Die Parolen im Vorwort sind nur eine Alibi. In: DIE WELT vom 7.2.1979. Vgl. schon vorher Giselher Stark (1966): Wir sprechen noch eine Sprache. Was Institute in West und Ost herausfanden. In: Der Telegraph (Berlin-West) vom 8.4.1966

42 WDG (1964ff.): Wörterbuch der deutschen Gegenwartssprache, hg. v. R. Klappenbach und W. Steinitz. 6 Bände, 1. Aufl. Berlin (Ost)

Duden-Wörterbuch (1976ff.): DUDEN - Das große Wörterbuch der deutschen Sprache in 6 Bänden, hg. v. G. Drosdowski u.a., Mannheim, Wien, Zürich 
Die Sprache bildet den vielleicht wichtigsten Träger der kulturellen Tradition, die von einer Generation auf die nächste übertragen wird. ${ }^{43}$

Wichtiges $\mathrm{zu}$ einem realistischeren, nüchternen, gleichwohl farbigen und widersprüchlichen Bild der DDR trugen auch die Berichte und Bücher der in der DDR akkreditierten Korrespondenten bei; mehrere sind als Taschenbücher erschienen. ${ }^{44}$ Einige Konstanten wies aber auch das neue, differenzierte Bild der DDR auf: Immer wieder und gewiß zu recht wurde hingewiesen auf die große Kluft zwischem angestrengtem, kontrolliertem, von allseitigem Selbstlob und lautstarkem Leistungsansporn geprägtem öffentlichen Leben und dem privaten. Günter Gaus prägte den Begriff "Nischengesellschaft"45. Sprachlich entspräche dem eine bemerkenswerte Fähigkeit der DDR-Bürger zum Umschalten zwischen öffentlichem und nichtöffentlichem Sprachregister. ${ }^{46}$ Während nun das öffentliche oder offizielle Sprachregister eine starke Sonderentwicklung nehme, weise das private - ebenso übrigens auch die anspruchsvolle Literatur - nur eine geringe Sonderentwicklung auf, sie werde überall verstanden.

In den achtziger Jahren öffnete sich die Diskussion hier auch einigen Gegenargumenten. In dem Maß, in dem die DDR kein Feindbild mehr war, erschien vieles als gleich oder ähnlich, was es nicht ist. So hört man bei Hinweisen auf sprachliche (und sachliche) DDRBesonderheiten z.B. oft die Entgegnung "Das ist doch bei uns genauso" - (meist stimmt sie nicht).

Auf der anderen Seite wird die Annahme, die Verständigung im nichtöffentlichen Bereich funktioniere problemlos, infragegestellt mit dem Hinweis, daß dies nur deshalb und nur so lange gelte, als die DDR-Bevölkerung in der Lage und weiterhin willens sei, ihren Sprachgebrauch in unseren oder in gemeindeutschen zu transferieren.

Bundesministerium für innerdeutsche Beziehungen (1974): Materialien zum Bericht zur Lage der Nation, Bonn, S. 73

Vgl. die Zusammenstellung bei M.W. Hellmann in: Mitteilungen des Instituts für deutsche Sprache 11, Mannheim 1985, Anm. 8 (S. 88). Zu ergänzen ist noch: Bussiek, H (1984): Die real existierende DDR. Neue Notizen aus der unbekannten deutschen Republik. (= Fischer Tb. Nr. 4246), Frankfurt

Gaus, G. (1986): Wo Deutschland liegt (=dtv Allgem. Reihe Nr. 1056)

46 Neben vielen anderen vgl. Eva Windmöller (1980): Leben in der DDR, Hamburg, darin Kapitel 12: "Die Kunst der doppelten Zunge" (S. 168 - 180). Mit Bezug darauf M.W. Hellmann (1978): Sprache zwischen Ost und West - Uberlegungen zur Wortschatzdifferenzierung zwischen BRD und DDR und ihre Folgen. In: Kühlwein / Radden (Hg.): Sprache und Kultur..., Tübingen, S. 15 - 54, hier S. 27 f. 
Denn diesen Transfer erbringen nämlich die DDR-Gesprächspartner fast allein. Dazu Manfred Ackermann, damals Mitglied der Ständigen Vertretung der Bundesrepublik in der DDR:

Die Menschen dort drüben empfinden die Teilung in ganz strengem Maße als politisch verfügt. Das heißt, sie haben sie innerlich nicht akzeptiert, und sie wehren sich auch sprachlich dagegen. Und da fast alle sprachlichen Vorgaben politischen Ursprungs sind, ist auch die Abwehrhaltung gegen solche Vorgaben ziemlich stark ausgeprägt. Das übernimmt man oberflächlich. Da engagiert man sich auch psychisch überhaupt nicht, das wird dann einfach abgehaspelt, aber wenn das nicht mehr verlangt wird, dann redet man eben anders.

Mein Eindruck ist, wenn wir Deutsche in Ost und West, in Bundesrepublik und DDR uns trotzdem fast nahtlos verstehen können, diese Leistung wird von den Bürgern der DDR erbracht, nicht von uns. Denn die DDRBürger, die in ihrem Staat schon in einer gewissen Doppelheit leben zwischen öffentlicher und privater Sprache, die leben auch in der Doppelheit von Westsprache und Ostsprache, von Westbegriffen und Ostbegriffen, und die können das übersetzen, was wir manchmal gar nicht können.

Die DDR-Bürger [...] sind in hohem MaB bereit, eine zusätzliche sprachliche Leistung oder denkerische Leistung zu bringen, indem sie für sich selbst, aber auch für uns, ihr Regime, ihren Sprachgebrauch, auch den Jargon übersetzen, wenn wir es nicht mehr verstehen. ${ }^{47}$

Der BRD-Bürger dagegen hat, so scheint mir, in der Regel die DDR (und ihre Bürger) aus dem nationalen Wir-Bewußtsein ausgeklammert und ihnen einen Status wie vielleicht Österreich (oder weniger) zugewiesen; dies zeigt sich auch im Gebrauch des Wortes "deutsch" und "Deutschland". 48

In der DDR hatte sich in den achtziger Jahren eine neue Entwicklung angebahnt, die letztlich die Abgrenzungspolitik zumindest abschwächte. Erich Honecker fand mehrfach überraschende nationale Töne, einen z. B. aus Anlaß der Nachrüstungsdebatte in einem Brief an Bundeskanzler Kohl 1983:

Ein atomwaffenfreies Europa ist letzten Endes das Ziel der europäischen Völker. Wir schließen uns im Namen des deutschen Volkes dem an. ${ }^{49}$

47 Ackermann, W. (1986): Podiumsdiskussion. In: Debus / Hellmann / Schlosser (Hg.) Sprachliche Normen und Normierungsfolgen in der DDR, Hildesheim, Berlin, New York, S. $302-303$

Zum Begriff "Deutschland" vgl u.a. H. Berschien (1974): Deutschland - ein Name im Wandel. (= Analysen und Perspektiven Sonderreihe Bd. 1), München, Wien

Erich Honecker: Brief an Bundeskanzler Helmut Kohl vom 9.Oktober 1983, in: Neues Deutschland vom $\mathbf{1 0 . 1 0 . 8 3}$ 
Seit Ulbricht hat kein führender SED-Politiker mehr "im Namen des deutschen Volkes" gesprochen.

Die DDR bemühte sich energisch, große Gestalten der deutschen bzw. preußischen Geschichte aus dem Kerker der ideologischen Verdammung in das helle Licht der nationalen Erbepflege zu heben - so Luther, den Preußenkönig Friedrich, sogar den Junker Bismarck nach dem Grundsatz: "Alle positiven Entwicklungslinien der deutschen Geschichte finden letztlich ihre Erfüllung in der sozialistischen DDR".

1982/83 greift diese neue Linie nun auch auf die Auffassung von Sprache über. Der Leipziger Sprachgermanist Wolfgang Fleischer, berühmt durch eine Reihe ausgezeichneter Hochschullehrbücher, hielt 1983 ein kurzes Referat auf einer Fachtagung, das folgendermaßen schloß:
Alles in allem: Es ist ein ProzeB im Gange; eine terminologische Fixierung, die über die Benennung "die deutsche Sprache in der DDR" hinausgeht, halte ich gegenwärtig für nicht angebracht. Im übrigen bezweifle ich, daB eine weitgehende Differenzierung oder Verselbständigung der deutschen Literatursprache in der DDR wünschenswert wäre [...]
[Die sehr unterschiedlichen sprachlichen Besonderheiten in der DDR] sind jedoch nicht DDR-isolationistisch zu sehen. Sie knüpfen an Benennungs- traditionen der Arbeiterklasse an, die sich im Deutschen in der Vergan- genheit in ne rhal b einer staatlichen Kommunikationsgemeinschaft mit antagonistischer Klassenstruktur bereits entwickelt hatten. ${ }^{50}$

Das war sensationell, bedeutete es doch nicht nur das Ende der VierVarianten-These, sondern auch eine Abwendung von den implizit unterstellten Bemühungen, die Differenzierung und Verselbständigung der Sprache in der DDR noch weiter zu treiben; dies sei "nicht wünschenswert".

Fleischer erhielt die Möglichkeit, sein neues Konzept in einer Arbeitsgruppe $\mathrm{zu}$ verwirklichen, die bald auf mehr als 12 Linguisten (fast alles Schüler von Fleischer) anwuchs; Ende 1987 legte Fleischer nun das Ergebnis ihrer 3 1/2jährigen Arbeit vor, die "Kollektiv-Monographie" (wie es drüben heißt) "Wortschatz der deutschen Sprache in der DDR". Dort heißt es im Vorwort:

Der propagandistische Mißbrauch, der von seiten bestimmter Kreise in der BRD mit Benennungen wie "deutsche Sprachgemeinschaft" getrieben wird, ist noch kein Grund, solche Benennungen als unzutreffend anzusehen. [...]

50 Fleischer, W. (1983): Die deutsche Sprache in der DDR. Grundsätzliche Uberlegungen zur Sprachsituation. In: Linguistische Studien H. 111, S. 258 - 275 
Die Herausbildung der DDR, eines selbständigen sozialistischen Staates, ist eine historische Tatsache, die Verwendung der deutschen Sprache als Staatssprache in verschiedenen Staaten ist eine andere historische Tatsache. [...] Im Unterschied zu historischen Prozessen in der Vergangenheit, die sich unter anderen Bedingungen vollzogen haben, ist die Selbstidentifizierung der sich in der DDR entwickelnden sozialistischen deutschen Nation nicht an eine eigene, besondere Sprache gebunden. [...] Die in der DDR erarbeiteten Kodifikationen sprachlicher Normen auf den verschiedenen Ebenen lassen keine weitgehende Normdivergenzen erkennen. Sie können im Gegenteil als repräsentativ für die deutsche Literatursprache (Standardsprache) der Gegenwart angesehen werden. [...] Die Frage nach der deutschen Sprache in der DDR ist demnach nicht prinzipiell einzuengen auf die 3-4 Jahrzehnte, die seit der offiziellen Gründung unserer Republik vergangen sind. Die Entwicklung der deutschen Sprache ist ein Element des Geschichtsprozesses, der schließlich zur Gründung des ersten sozialistischen deutschen Staates geführt hat.

Wenn mit der Gründung der DDR "ein neues Kapitel in der Geschichte des deutschen Volkes aufgeschlagen wurde" - [...], so hat sich im Zusammenhang damit auch für die sprachliche Kommunikation und die Entwicklung der deutschen Sprache eine neue Situation ergeben. ${ }^{51}$

Die Autoren beschäftigen sich fast ausschließlich mit den Besonderheiten der DDR, vergleichen nicht oder kaum und greifen den westdeutschen Gebrauch kaum an.

In der Zwischenzeit hatten westdeutsche Sprachgermanisten den Faden neu aufgegriffen. Der Sammelband von Debus/Hellmann/Schlosser von 1986 beschäftigt sich überwiegend mit DDR-Sprachgebrauch, jedoch in mehreren Beiträgen auch vergleichend. Wie spannend die am Sammelband Beteiligten diese neue DDR-Entwicklung fanden, geht aus dem Schlußwort hervor:

Wir können uns leicht darauf verständigen, daß wir diese neue Entwicklung begrüBen als ausgesprochen produktiv für die DDR-Linguisten selbst und hilfreich für uns für eine mögliche Kommunikation mit den DDR-Linguisten [...]

Aber dabei sollte es nicht bleiben. In jedem zweiten Ost-West-Kommuniqué steht heute - erfreulicherweise - ein Satz wie "Wir haben eine gemeinsame Verantwortung für den Frieden in Europa". Wir haben auch, das ist unstreitig und in Verträgen gesichert, eine gemeinsame Verantwortung für den Umweltschutz, den Gesundheitsschutz, einen reibungslosen Verkehrsfluß und vieles andere - manchmal sogar wird anerkannt: für

51 Fleischer, W. (1987): Wortschatz der deutschen Sprache in der DDR, Leipzig, (aus dem Vorwort S. 14 und 15) 
unser gemeinsames kulturelles Erbe. Vielleicht öffnet sich allmählich der Weg für die Erkenntnis: auch für unsere gemeinsame Sprache. ${ }^{\mathbf{5 2}}$

Gleichzeitig mit Fleischers Band erschien in Bern ein Tagungsband, der auch eine Podiumsdiskussion dokumentiert. An ihr hatten, neben Österreichern und natürlich Schweizern, Peter von Polenz für die BRD und Wolfdietrich Hartung, prominenter Linguist aus dem ZISW in Ostberlin, teilgenommen. Unter Berufung auf den australischen Germanisten Michael Clyne ${ }^{53}$ diskutierten sie den neuen Begriff "plurizentrisch" - "Plurizentrismus"54.

[Wolfdietrich Hartung: ...] Es hat sich ein besonderer Wortschatz herausgebildet, es gibt nur ein paar Textmuster wahrscheinlich. Es gibt wahrscheinlich [...] verschiedene Bewertungen von Kommunikationsstrategien und ein unterschiedliches Reflektieren über die kommunikative Wirklichkeit. Aber das alles erfolgt im Rahmen einer einheitlichen Sprache, der deutschen Sprache. Ich wäre sehr vorsichtig mit der Charakterisierung dieser Besonderheit als nationale Variante. Es ist die deutsche Sprache mit ein paar Besonderheiten im Rahmen einer einheitlichen Sprache [...]

Sprache [ist] immer bezogen [...] auf einen ganz bestimmten Erfahrungshintergrund, [...] und wenn dieser Erfahrungshintergrund durch eine staatlich organisierte, umgrenzte Kommunikationsgemeinschaft geprägt ist, kann es da natürlich Prägungen geben, die zwar sehr schwer merklich und auch relativ schwer in Wörterbüchern darstellbar sind, die aber insgesamt für die Entwicklung der Sprachgemeinschaft auf längere Sicht nicht ganz unwichtig sind. [S. 58]

[Peter von Polenz:] Die Bevölkerung der Bundesrepublik Deutschland bildet seit 1945 eine eigene Kommunikationsgemeinschaft [...], deren Sprachpraxis stark von einer eigenen Politik dieses Staates geprägt und verändert worden ist. [...] Sprachliche Besonderheiten der Bundesrepublik Deutschland lassen sich also mindestens in gleichem Umfang auflisten wie dies in der Forschung bisher fast nur für die anderen deutschsprachigen Staaten getan worden ist. [...] Dies berechtigt uns zu einem Ansatz einer westdeutschen oder bundesdeutschen Variante der deutschen Sprache, aber in dem Sinne, daB wir hier nur bestimmte Funktionalstile, bestimmte Situationsregister, bestimmte Textsortenstile meinen, die man als Staats-

52 Aus dem Schlußwort. In: Debus / Hellmann / Schlosser (1986) (Hg.): Sprachliche Normen und Normierungsversuche in der DDR, S. 295

Clyne, M. (1984): Language and Society in the German-speaking Countries. Cambridge University Press, Cambridge, London, Melbourne, Sidney

Nach Abschluß des Manuskripts erscheint Peter von Polenz: 'Binnendeutsch' oder plurizentrische Sprachkultur? Ein Plädoyer für Normalisierung in der Frage der 'nationalen' Varietäten. In: Zeitschr. f. Germanistische Linguistik, 1988, Heft 2. Dazu die Stellungnahme von Manfred W. Hellmann (1988): 'Binnendeutsch' und 'Hauptvariante Bundesrepublik' - Zu Peter von Polenz' Kritik an Hugo Moser. In: ZGL 1988, H. 3 
bürger der Bundesrepublik und als Mitglied ihrer Kommunikationsgemeinschaft beherrschen muB, um als solche akzeptiert zu werden [...] [S. 58f.]

[...] Nun gibt es die neuere Auffassung [...], daB die Beziehungen zwischen den deutschsprachigen Staaten plurizentrisch sind. Das heißt also, daB die deutsche Sprache als Kultureinheit mehrere Zentren hat, die gegenseitig Einfluß aufeinander ausüben [...].

[...] die Behauptung [die Sprachvariante der BRD sei die] "Hauptvariante", stimmt nicht überein mit der tatsächlichen Auffassung der meisten Deutschen. [...] Ich glaube, es ist heute für die meisten Menschen [...] eine Selbstverständlichkeit, daB die anderen deutschsprachigen Staaten ganz gleichwertig ihren Beitrag zur deutschen Sprache und Literatur als Kultureinheit leisten, und ich glaube, es gibt sehr viele bei uns, die sich darüber freuen. [S. 64f.]

[Wolfdietrich Hartung:] Also mit der These des Plurizentrismus bin ich völlig einverstanden. Die ganze historische Entwicklung ist glaube ich gar nicht anders verständlich, als wenn man eine plurizentrische Basis der Sprachentwicklung im deutschen Sprachraum annimmt.[S. 66] ${ }^{55}$

Hier sind wir nun am vorläufigen Ende angelangt.

\section{Resümee und Ausblick}

Ausgehend von der zunächst gemeinsamen Sorge um die Einheit und Reinheit der deutschen Sprache in den ersten acht Jahren nach dem Krieg entwickelten sich die Einstellungen zu dem Phänomen der sprachlichen Ost-West-Differenzierung in entgegengesetzte Richtung:

In der Bundesrepublik befürchtete man vielfach eine Sprachspaltung, verursacht durch die "Begriffsverdrehungen", das "Moskauderwelsch" der Partei, der SED-Funktionäre. In der DDR wehrte man sich in einer gereizten Verteidigungshaltung mit Gegenvorwürfen: in Westdeutschland werde die Spracheinheit gefährdet, die DDR entwickele sie positiv weiter, sie garantiere die Einheit der Nation und die Reinheit der Sprache.

Zwischen 1967 und 69 änderte sich das Bild: Die DDR ging auf Abgrenzungskurs, bestritt bestehende Gemeinsamkeiten und konstatierte die Auflösung der Einheit von Sprache und Kultur, während die Publizistik der BRD, z.T. gestützt von Argumenten der

55 VIII. Internationale Deutschlehrertagung Bern (1987): Ziele und Wege des Unterrichts in Deutsch als Fremdsprache. Tagungsbericht, hg. von R. Zellweger. Staatl. Lehrmittelverlag Bern, S. 58 - 66 
Sprachwissenschaftler, die sprachliche Einheit als verbindendes Element betonte. Ab 1970 - Verhandlungspolitik und Abgrenzungspolitik - wurden beide politischen Positionen linguistisches Programm, für die Linguistik der DDR manifestiert in der "Vier-VariantenThese", in der BRD in der wiederaufgegriffenen Methapher vom "einigenden Band der deutschen Sprache".

Während die Sprachwissenschaft in der BRD in den achtziger Jahren begann, die Ergebnisse der Wortschatzforschung zu relativieren und das "einigende Band" zu problematisieren, entdeckte die DDR ein erneutes Interesse an nationaler Kontinuität, sie entdeckte auch neue Gemeinsamkeiten; sie verabschiedete unauffällig die "Vier-Varianten-These".

Somit hat die doppelte Wende nun zu einem unerwarteten Resultat geführt: Beide Seiten sind sich - wie auch die Politiker - in bestimmten Punkten einig, sie verwenden die gleichen Formeln: Es gibt eine deutsche Sprache mit ihren Besonderheiten in der BRD, in der DDR usw. Diese prägen durchaus auch das sprachliche Erscheinungsbild ihrer jeweiligen Kommunikationsgemeinschaft, aber in engstem Zusammenhang mit der gemeinsamen geschichtlichen Herkunft. "Plurizentrismus" könnte das neue Denkmodell heißen.

Vielleicht ist dies nur ein Moment, bevor die Drehung wieder beginnt. Ansätze zu neuer Drehung gäbe es. Die DDR könnte ihre Anstrengungen in Richtung deutsch-deutscher Gemeinsamkeiten verstärken, aus eigenem Interesse, aber auch um die BRD bei ihren Verbündeten in Schwierigkeiten zu bringen; sie könnte versuchen, hier die argumentative Führung zu übernehmen. Die Intensität der selektiven Erbe-Aneignung in der DDR spricht für diese Option. Das würde voraussichtlich in der BRD die Tendenz verstärken, die Unterschiede zu betonen.

Auch dafür gibt es Ansätze:

1. Allgemein akzeptiert bei uns ist wie gesagt die Annahme, es gebe in der DDR eine beträchtliche Kluft zwischen offiziellem und privatem Sprachregister, viel größer und tiefgreifender als bei uns. Darauf könnte man leicht eine Hypothese aufbauen (ähnlich wie früher): die Deutschen verstehen sich ja untereinander. Nur die SED, das System und seine Ausdrucksweise vom Volk nicht akzeptiert - stören furchtbar. Eine solche - zu einfache - Hypothese wäre natürlich hochgradig konfliktär. 
2. Unsere Medien bringen verdächtig oft Glossen der Art, daß angebliche oder wirkliche Albernheiten des DDR-Sprachgebrauchs als typisch dargestellt werden - Tenor (salopp gesagt): "So etwas von Verhunzung! Typisch DDR - kommt bei uns nicht vor!"56 Allgemeiner: Es läßt sich eine Interessenlage denken, die es für die BRD nahelegen könnte, die DDR als schon recht weit in Richtung Sozialismus abgedriftet darzustellen. Dafür gäbe es psychologische Ansatzpunkte: Das (amtlich geförderte ${ }^{57}$ ) Selbstverständnis der meisten BRD-Deutschen, sie allein seien deutsch, seien Deutschland, könnte die DDR immer fremder oder doch gleichgültiger erscheinen lassen. Das ist Spekulation natürlich, aber eine wohl doch nicht ganz abwegige.

Daraus ließe sich eine neue Runde von Polemiken, auch sprachwissenschaftlichen, entwickeln - wenn man will. Man kann aber auch anders wollen.

Vielleicht ist es doch mehr als nur ein Moment der Ruhe, bevor der Kreisel sich zur nächsten Wende dreht. Vielleicht gelingt es wirklich, die deutsche Sprache in den Katalog gemeinsamer Verantwortung aufzunehmen. Das würde gemeinsame oder doch immerhin abgestimmte Forschungsvorhaben möglich machen in ganz anderem $\mathrm{Maß,}$ als das bisher vorstellbar schien. $\mathrm{Zu}$ tun gäbe es mehr als genug.

Ich schließe mit einem Zitat von Christa Wolf:

Auch heute wachsen Kinder auf in den beiden deutschen Staaten. Fragen wir uns denn ernst genug: Wie sollen die, wenn sie groß sind, miteinander reden? Mit welchen Wörtern, in was für Sätzen, und in welchem Ton? ${ }^{58}$

56 Als Beispiel von vielen vgl. "Wort-Kluft Ost-West? Auf erfundene DDR-Wörter reingefallen". In: Sprachreport (IDS), Nr. 4, 1986. Gegen solche Tendenzen wendet sich z.B. auch Horst Dieter Schlosser (Univ. Frankfurt), jedoch scheint der Erfolg solcher Bemühungen leider eher gering.

Ich beziehe mich auf die amtlichen "Bezeichnungsrichtlinien" von 1974, die den Gebrauch der Abkürzung "BRD" ablehnen und - sofern ohne MiBverständnisse möglich - die Bezeichnung "Deutschland" (und "deutsch") statt "Bundesrepublik (Deutschland)" empfehlen

Wolf, C. (1980): Ein Satz. Rede zur Verleihung des Bremer Literaturpreises am 26.1.1978. In: Lesen und Schreiben. Neue Sammlung. Darmstadt, Neuwied, S. 105 


\section{Literatur}

Ackermann, M. (1986): Podiumsdiskussion. In: Debus/Hellmann/Schlosser (Hg.): Sprachliche Normen und Normierungsfolgen in der DDR, Hildesheim, Berlin, New York 1986, S. $302-303$

Ahrends, M. (1986): Trabbi, Telespargel und Tränenpavillon - Das Wörterbuch der DDR-Sprache (=Heyne Taschenbuch Nr. 6754), München 1986

Becker, H. (1956): Sieben Sprachbriefe zur Gegenwart. Eine gesellschaftliche Sprach- und Denklehre, Halle 1956

Berschin, H. (1974): Deutschland - ein Name im Wandel (=Analysen und Perspektiven, Sonderreihe Bd. 1), München, Wien 1974

Betz, W. (1962): Zwei Sprachen in Deutschland? In: Merkur 16/9 1962, S. 873 - 879. Nachdruck in: Handt (1964), S. 155 163

Borée, K.-F. (1952): Die Sprachentartung in der Sowjetzone. In: PZArchiv 3/2, 1952, S. 23 - 24

Brandt, W. (1970): Zur Lage der Nation. Bonn 1970

Bundesministerium für gesamtdeutsche Fragen $\left({ }^{2} 1954,{ }^{8} 1963\right)$ : SBZ von A bis Z. Bonn 1954, 1963

Bundesministerium für innerdeutsche Beziehungen (1972): Bericht der Bundesregierung und Materialien zur Lage der Nation, Bonn 1972

Bundesministerium für innerdeutsche Beziehungen (1974): Materialien zum Bericht zur Lage der Nation, Bonn 1974

Bussiek, H. (1984): Die real existierende DDR. Neue Notizen aus der unbekannten deutschen Republik. (=Fischer $\mathrm{Tb}$. $\mathrm{Nr}$. 4246), Frankfurt 1984

Clyne, M. (1984) Language and Society in the German-speaking Contries. Cambridge University Press, Cambridge, London, Melbourne, Sidney 1984

Debus, Fr. / Hellmann, M. W. / Schlosser, H. D. (Hg.) (1986): Sprachliche Normen und Normierungsfolgen in der DDR. Hildesheim, Berlin, New York 1986

Dieckmann, W. (1967): Kritische Bemerkungen zum sprachlichen Ost-West-Problem. In: Zeitschrift für deutsche Sprache 23/3 1967, S. 136 - 165 
Dittmar, P. (1979) Die Parolen im Vorwort sind nur ein Alibi. In: DIE WELT vom 7.2.1979

Fleischer, W. (1983): Die deutsche Sprache in der DDR. Grundsätzliche Überlegungen zur Sprachsituation. In: Linguistische Studien Reihe A, H. 111, 1983, S. 258 - 275

Ders. (1987): Wortschatz der deutschen Sprache in der DDR. Leipzig 1987

Gaus, G. (1986): Wo Deutschland liegt. (=dtv Allgem. Reihe Nr. 1056), 1986

Gernentz, H.-J. (1965): Droht dem Deutschen die Gefahr einer Spaltung in zwei Sprachen? In: jezyky obce szkole (Fremdsprachen in der Schule) Warschau, 2/2, 1965, S. $69-80$

Ders. (1967): Zum Problem der Differenzierung der deutschen Sprache in beiden deutschen Staaten. In: Weimarer Beiträge $13 / 3,1967$, S. $463-468$

Haefs, H. (1956): Gefährliches "Parteichinesisch". Verhängnisvolle Sprachspaltung zwischen West und Ost. In: Das Parlament 15.8.1956, S. 9 (Beilage "Sprache - Grundlage staatlichen Lebens")

Handt, F. (Hg.) (1964): Deutsch - Gefrorene Sprache in einem gefrorenen Land? Polemik, Analysen, Aufsätze. Berlin 1964

Hellmann, M. W. (1976): Bibliographie zum öffentlichen Sprachgebrauch in der Bundesrepublik Deutschland und in der DDR (=Sprache der Gegenwart Bd. 16), Düsseldorf 1976

Ders. (1978): Sprache zwischen Ost und West - Überlegungen zur Wortschatzdifferenzierung zwischen BRD und DDR und ihren Folgen. In: W. Kühlwein / G. Radden (Hg.): Sprache und Kultur: Studien zur Diglossie, Gastarbeiterproblematik und kulturellen Integration. Tübingen 1978, S. $15-54$

Ders. (1980): Deutsche Sprache in der Bundesrepublik Deutschland und in der Deutschen Demokratischen Republik. In: Lexikon der Germanistischen Linguistik, 2. Aufl. Tübingen 1980, S. 519 - 527

Ders. (1984): Ost-West-Wortschatzvergleiche (=Forschungsberichte des Instituts für Deutsche Sprache, Mannheim, Bd. 48), Tübingen 1984

Ders. (1985): Bemerkungen zur Entwicklung und zur gegenwärtigen Lage des Arbeitsgebietes "Ost-West-Sprachdifferenzie- 
rung". In: Mitteilungen 11 des Instituts für deutsche Sprache, Mannheim 1985, S. 76 - 93

Ders. (1986): Wort-Kluft Ost-West? Auf erfundene DDR-Wörter reingefallen. In: Sprachreport (IDS) 4/86

Ders. (1988): 'Binnendeutsch' und 'Hauptvariante Bundesrepublik' Zu Peter von Polenz' Kritik an Hugo Moser. In: Zeitschr. f. Germanistische Linguistik 1988, H. 3

Herrman, E. M. (1958): Die Sprache als Waffe. In: Hessische Blätter für Volksbildung $8 / 3,1958$, S. $135-140$

Heyden, G. (1968): Manipulation. Die staatsmonopolistische Bewußtseinsindustrie. Berlin (Ost) 1968

Höppner, J. (1964): Widerspruch aus Weimar. In: Handt, Fr. (1964), S. $143-151$

Honecker, E. (1973): Rede auf der 9. Tagung des Zentralkomitees 1973. In: Neues Deutschland vom 29.5.1973

Ders. (1983): Brief an Bundeskanzler Helmut Kohl vom 9. Oktober 1983. In: Neues Deutschland vom 10.10.1983

Ihlenburg, K.-H. (1964): Entwicklungstendenzen des Wortschatzes in beiden deutschen Staaten. In: Weimarer Beiträge 10/3, 1964, S. 372 - 397

Kahn, S. (1974): Nation und Sprache. In: Weltbühne Nr. 53 vom 31.12.1974 (Berlin-Ost)

Kinne, M. / Strube-Edelmann, B. (1981): Kleines Wörterbuch des DDR-Wortschatzes, 2. Aufl., Düsseldorf 1981

Klemperer, V. (1952): Unsere Sprache - ein einigendes Band der Nation. In: Die neue Schule, J. 8/52, S. 4 - 5

Ders. (1954): Zur gegenwärtigen Sprachsituation in Deutschland. (=Vorträge zur Verbreitung wissenschaftlicher Kenntnisse, Bd. 17), Berlin (Ost) 1954 (1. Aufl. 1952)

Ders. (1955): Verantwortung für die Sprache. In: Neue deutsche Literatur, Jg. 3, H. 3, 1955, S. $122-126$

Ders. (1969): "LTI" - Die unbewältigte Sprache. Aus dem Notizbuch eines Philologen. dtv 3. Aufl. 1969 (1. Auf1. 1947)

Köhler, A. (1954): Deutsche Sprache in östlicher Zwangsjacke. (=Vortrag im deutschen Sprachverein Berlin, 4.12.53), Berlin (West) 1954

Koepp, Fr. (1955): "Sowjetdeutsch" - Die Sprache als Opfer und Werkzeug der Sowjetisierung. In: Akademische Blätter $57 / 3,1955$, S. $41-46$ 
Korlén, G. (1959): Zur Entwicklung der deutschen Sprache diesseits und jenseits des eisernen Vorhangs. In: Deutschunterricht für Ausländer, Jg. 9, 1959, S. 138 - 153; Nachdruck in: Handt (Hg.) (1964), S. 123 - 143

Korntner, B. (1959): "Sowjet-Deutsch" - Die Sprache als politisches Kampfmittel der Kommunisten. In: Die Orientierung (Pfaffenhofen), Beiheft Nr. 12 o.J. (1959)

Lerchner, G. (1976): Nationalsprachliche Varianten. In: Forum 3/76

Luft, Fr. (1958): Wenn das Wörterbuch zur Parteifibel wird. Der Duden links und rechts der Zonengrenze. Wir sprechen schon zweierlei Deutsch. In: DIE WELT vom 5.4.1958

Maeder, H. (1964): Sprache und Totalitarismus. In: Moser (1964): Das Aueler Protokoll, S. 13 - 23

Moser, H. (1961): Die Sprache im geteilten Deutschland. In: Wirkendes Wort $11 / 1,1961$, S. 1 - 21

Ders. (1962): Sprachliche Folgen der politischen Teilung Deutschlands. (=Beiheft zum Wirkenden Wort Nr. 3), Düsseldorf 1962

Ders. (Hg.) (1964): Das Aueler Protokoll - Deutsche Sprache im Spannungsfeld zwischen Ost und West. Düsseldorf 1964.

Ders. (1971): Zum Problem der 'neutralen' Neuprägungen und Neubedeutungen im of fiziellen Sprachgebrauch der DDR. In: Schenk, S. (Hg.) u.a.: Et multum et multa - Festgabe für Kurt Lindner, Berlin/New york 1971, S. 249 - 255

Polenz, P. v. (1988): 'Binnendeutsch' oder plurizentrische Sprachkultur? Ein Plädoyer für Normalisierung in der Frage der 'nationalen' Varietäten. In: Zeitschrift für Germanistische Linguistik 1988, Heft 2

Richter, W. (1953): Zur Entwicklung der deutschen Sprache in der sowjetischen Besatzungszone. In: Europa-Archiv 8/21, 1953, S. 6053 - 6056

Riemschneider, E. G. (1964): Sprachliche Veränderung im Bereich der Landwirtschaft. In: Moser, H. (Hg.) (1964), S. 80 - 91

Roegele, O. B. (1959): Die Spaltung der Sprache. Das kommunistische Deutsch als Führungsmittel. In: Die politische Meinung 4/36, 1959, S. $48-60$

Schaeder, B. (1981): Deutsche Sprache in der BRD und in der DDR. Neuere Arbeiten und Ansichten über das sprachliche OstWest-Problem. In: Muttersprache, Jg. 91, H. 3/4, 1981, S. $198-205$ 
Schierbaum, H. (1964): Das Wort als politisches Instrument. In: Moser, H. (Hg.) (1964), S. 24 - 39

Staatsrat der DDR (1969): Entwurf eines Vertrages über die Herstellung völkerrechtlicher Beziehung zwischen der Deutschen Demokratischen Republik und der Bundesrepublik Deutschland. In: Neues Deutschland vom 18./19. Dezember 1969 , S. 1

Stark, G. (1966): Wir sprechen noch eine Sprache. Was Institute in West und Ost herausfanden. In: Der Telegraph (BerlinWest) vom 8.4.1966

Ulbricht, W. (1970): Rede auf der 13. Tagung des ZK der SED. In: Neues Deutschland 25. Jg., Nr. 164, 16. Juni 1970, S. 4

Weiskopf, F. C. (1955): "Ostdeutsch" und "Westdeutsch" oder über die Gefahr der Sprachentfremdung. In: Neue Deutsche Literatur $3 / 7,1955$, S. $79-88$

Windmöller, E. (1980): Leben in der DDR (=Goldmann Taschenbuch 11502), Hamburg 1980

Wolf, C. (1980): Ein Satz. Rede anläßlich der Verleihung des Bremer Literaturpreises am 26.1.1978. In: Lesen und Schreiben Neue Sammlung. Essays, Aufsätze, Reden. (= Sammlung Luchterhand Nr. 295). Darmstadt, Neuwied 1980, S. 100 $-105$

Zellweger, R. (Hg.) (1987): VIII. Internationale Deutschlehrertagung Bern: Ziele und Wege des Unterrichts in Deutsch als Fremdsprache - Sein Beitrag zur interkulturellen Verständigung. Tagungsbericht. Staatlicher Lehrmittelverlag Bern 1987 